\title{
Complete Remission of Metastatic HER2+ Oesophagogastric Junctional Adenocarcinoma under long-term Trastuzumab Treatment
}

\author{
Tobias Gutting ${ }^{1}$, Nadine Schulte ${ }^{1}$, Sebastian Belle ${ }^{1}$, Johannes Betge ${ }^{1}$, Nicolai Härtel ${ }^{1}$, Jürgen Wilke ${ }^{2}$, Jürgen Weers ${ }^{3}$, \\ Matthias P. Ebert ${ }^{1}$, Tianzuo Zhan ${ }^{1}$
}

1) Department of Medicine

II, University Hospital

Mannheim, Medical Faculty

Mannheim, Heidelberg

University, Mannheim

2) Institute of Pathology,

Ludwigshafen

3) Gastroenterologische

Schwerpunktpraxis,

Mannheim, Germany

\author{
Address for correspondence: \\ Tobias Gutting \\ Department of Medicine \\ II, University Hospital \\ Mannheim, Medical Faculty \\ Mannheim, Heidelberg \\ University, Mannheim \\ tobias.gutting@umm.de
}

\begin{abstract}
Metastatic gastric cancer (GC) and oesophagogastric junctional (OGJ) adenocarcinoma have a poor clinical outcome with a high worldwide burden of disease. A 65 -year old male patient with microcytic anemia was diagnosed with stage IV OGJ adenocarcinoma with multiple liver metastases. Immunohistochemical analysis revealed a high expression of HER2 (3+). Palliative chemotherapy with FLOT (oxaliplatin, 5-fluorouracil, leucovorin and docetaxel) in combination with trastuzumab was initiated. Due to severe adverse events, the therapy was de-escalated to trastuzumab monotherapy after six months of treatment. Initial restaging revealed partial response after the combination therapy of FLOT with trastuzumab. After reduction to trastuzumab monotherapy, the disease remained stable for two years until radiological complete response was observed. Trastuzumab monotherapy was continued for another two years to maintain complete response. Eleven months after the discontinuation of the therapy, no recurrence of the disease was detected. In conclusion, complete response can be achieved under trastuzumab monotherapy in exceptional responders.
\end{abstract}

Key words: junctional adenocarcinoma - oesophagogastric junction cancer - metastatic disease - HER2 status - trastuzumab - complete response.

Abbreviations: ADCC: antibody-dependent cell-mediated cytotoxicity; BC: breast cancer; CR: complete response; ESMO: European Society for Molecular Oncology; FcR: Fc receptor; GC: gastric cancer; HER2: human epidermal growth factor receptor 2; OGJ: oesophagogastric junctional; PR: partial response; SD: stable disease; TIC: trastuzumab-induced cardiotoxicity.

\section{INTRODUCTION}

In 2018, the global incidence of gastric cancer (GC) and oesophagogastric junctional (OGJ) adenocarcinoma was estimated to be more than $1,000,000$ cases [1]. While the incidence of gastric cancer has declined during the last decades, the number of cases of OGJ adenocarcinoma has increased over the same period. Surgery is still the backbone of a curative treatment approach and introduction of perioperative/ neoadjuvant chemotherapy further improved survival rates [1]. However, most cases of gastric and OGJ adenocarcinomas are diagnosed at a locally advanced or metastatic stage and survival rates remain poor. For a long time, treatment of metastatic gastric and OGJ adenocarcinoma has relied on combination chemotherapy. Starting in 2002, a series of clinical trials demonstrated that trastuzumab, an antibody that targets the human epidermal growth factor receptor 2 (HER2), alone or in combination with chemotherapy can prolong survival in patients with HER2+ breast cancer (BC). HER2 overexpression can be determined and scored by immunohistochemistry and fluorescence in situ hybridization. The prevalence of HER2 overexpression in GC and OGJ adenocarcinoma ranges from 12 to $22 \%$, varying by tumor location and type $[2,3]$, which is similar to BC $[4,5]$. The phase III ToGA trial, which included patients with HER2 + metastatic GC and OGJ adenocarcinoma, demonstrated a superior survival for trastuzumab plus chemotherapy versus chemotherapy alone and established trastuzumab as a new standard in stage IV disease with HER2 overexpression [6]. Here, we present a case of a patient with OGJ adenocarcinoma and liver metastases who achieved a durable complete response (CR) after an initial brief treatment 
with combined chemotherapy and trastuzumab, followed by a long treatment period with trastuzumab monotherapy.

\section{CASE REPORT}

In April 2013, a 65-year old Caucasian male, diagnosed with OGJ adenocarcinoma was admitted to our department for further staging and treatment. In a routine blood analysis, his general practitioner diagnosed a microcytic anemia which was refractory to oral iron substitution. Weight loss, nausea, dysphagia, abdominal pain or changes of defecation were negated by the patient. The patient was not aware of any cancer disease in his family. He stated to be a non-smoker with occasional moderate alcohol consumption. Physical examination revealed an ECOG 1 performance status and overweight $\left(1.73 \mathrm{~m}, 90 \mathrm{~kg}, \mathrm{BMI} 30.1 \mathrm{~kg} / \mathrm{m}^{2}\right)$ with no symptoms of major organ or mental dysfunctions. A repeated oesophagogastroduodenoscopy (Fig. 1) with tissue biopsies performed in our department confirmed the diagnosis of an OGJ adenocarcinoma.

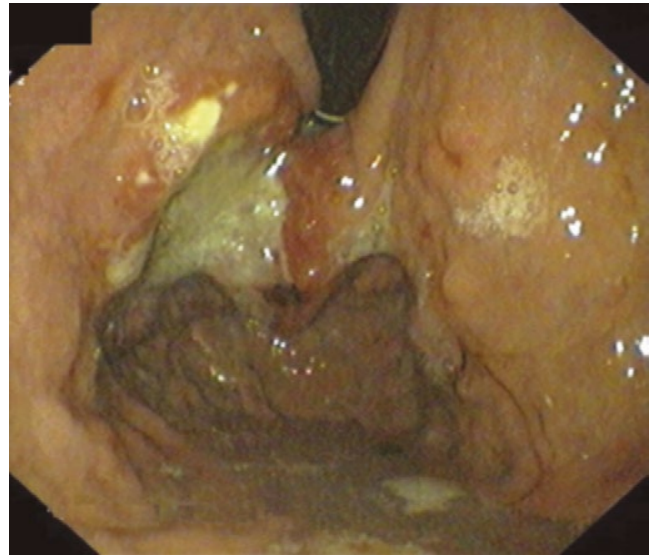

Fig. 1. Upper gastrointestinal endoscopy: ulcerated tumor located at the oesophagogastric junction.

Medical imaging combined with histopathological examination (Fig. 2) resulted in a moderately differentiated (G2) UICC stage IV intestinal type adenocarcinoma with the following TNM classification: cT3, cN2, cM1 (disseminated liver metastasis, Fig. 3). Immunohistochemistry revealed an amplification of HER2 with 3+ score (Fig. 4). Microsatellite analysis showed no microsatellite instability.

Treatment management is shown in Fig. 5. One month after diagnosis, palliative chemotherapy according to FLOT therapy regimen (bi-weekly oxaliplatin $85 \mathrm{mg} / \mathrm{m}^{2}$, leucovorin $200 \mathrm{mg} /$ $\mathrm{m}^{2}$, docetaxel $50 \mathrm{mg} / \mathrm{m}^{2}$, followed by 5 -FU $2600 \mathrm{mg} / \mathrm{m}^{2}$ as $24 \mathrm{~h}$ infusion) with additional trastuzumab ( $4 \mathrm{mg} / \mathrm{kg}$ body weight bi-weekly) was initiated. Treatment was discontinued after 3 cycles due to CTC grade 3 hand-foot syndrome and reduced to docetaxel and trastuzumab. First restaging after three months showed partial response (PR) to the treatment. Since CTC grade 3 dermal toxicity re-occurred, therapy was stopped 6 months after initial treatment. After a short period of recovery, treatment was re-initiated with trastuzumab as monotherapy, resulting in radiological stable disease (SD) in consecutive CT and MRI scans. Repeatedly carried out echocardiography examinations and follow-up visits demonstrated no adverse

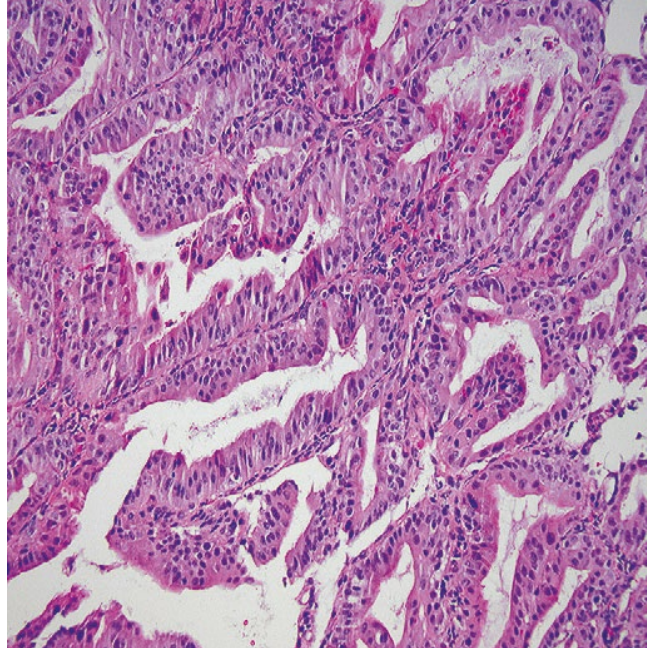

Fig. 2. Histopathology: moderately differentiated (G2) intestinal type adenocarcinoma (H\&E, 200x).

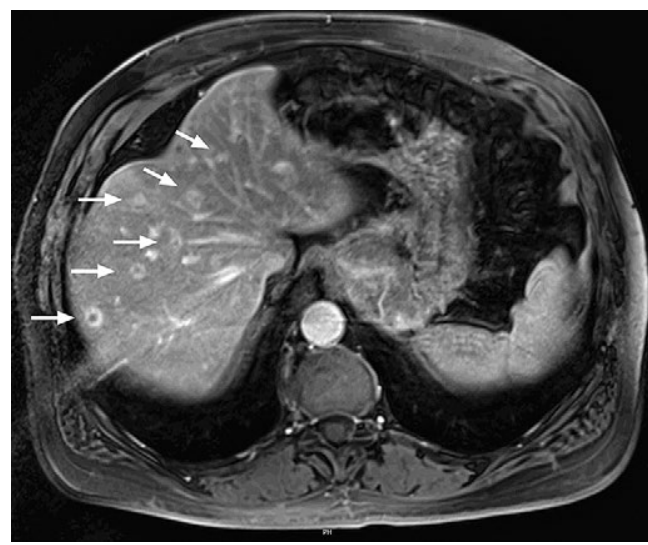

Fig. 3. MRI scan of the liver at diagnosis. Six liver metastases indicated by white arrows in $\mathrm{T} 1$ vibe sequences.

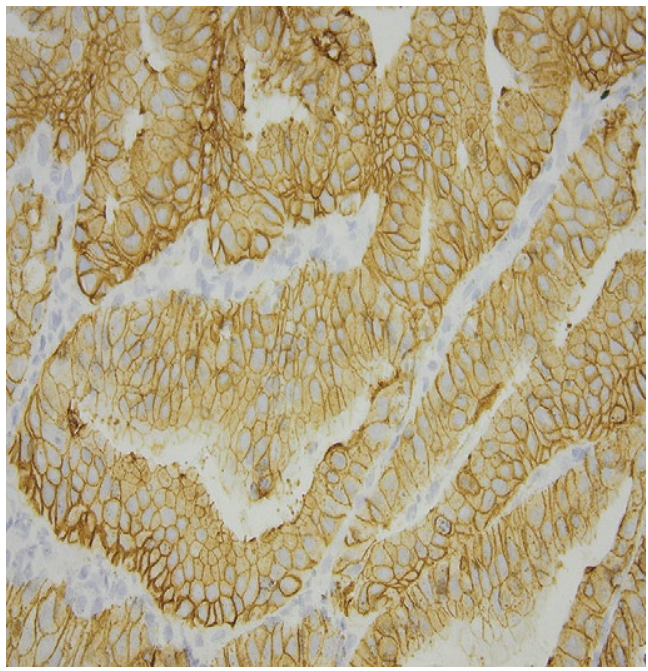

Fig. 4. Immunohistochemistry: HER2 positivity; HER2 3+ score (400-fold magnification).

effects of trastuzumab monotherapy. Twenty-three months after starting of trastuzumab monotherapy, the patient showed radiological CR with no detectable liver metastasis or enlarged lymph nodes. Oesophagogastroscopy performed three years 


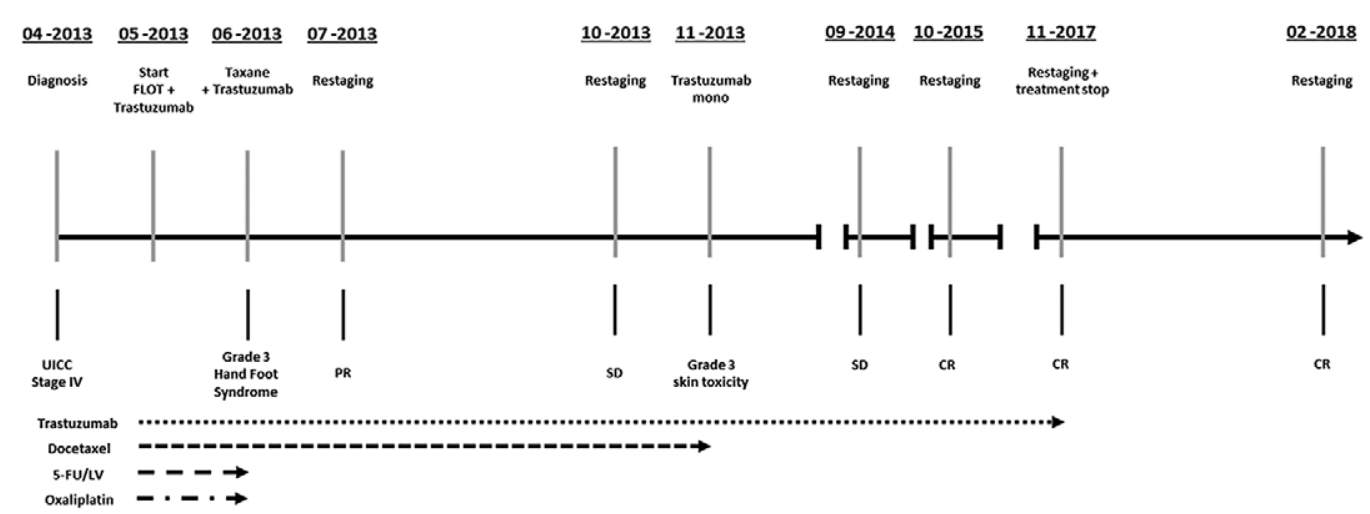

Fig. 5. The timeline is shown as an intermittent black line. Below every date, the clinical event is represented, beginning from the diagnosis until the last document staging. Occurrence of adverse events and results of staging examinations are shown under the timeline (PR: partial response; SD: stable disease, CR: complete response). The administered drugs and the respective durations of treatment are displayed as intermittent lines at the bottom.

after initial diagnosis showed no residual endoluminal tumor (Fig. 6). This disease status was maintained with trastuzumab monotherapy for more than 24 months. In agreement with the patient, we decided to discontinue treatment after four and a half years under close radiological surveillance. Eleven months after discontinuation of trastuzumab monotherapy, repeated CT scans showed no reoccurrence of the disease (Fig. 7).

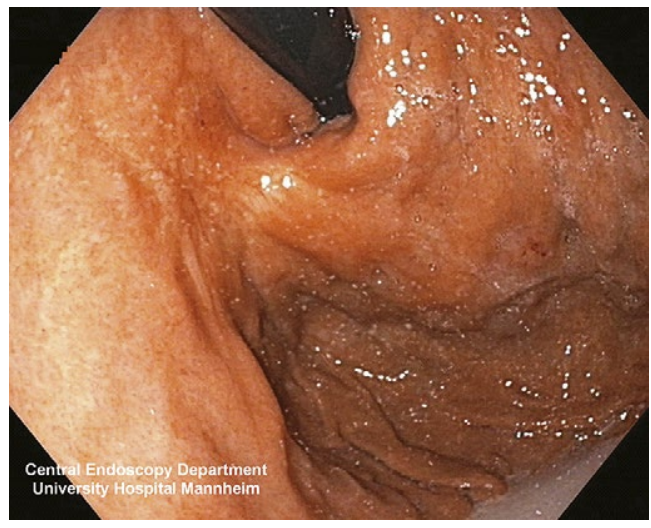

Fig. 6. Upper gastrointestinal endoscopy: no macroscopically detectable tumor.

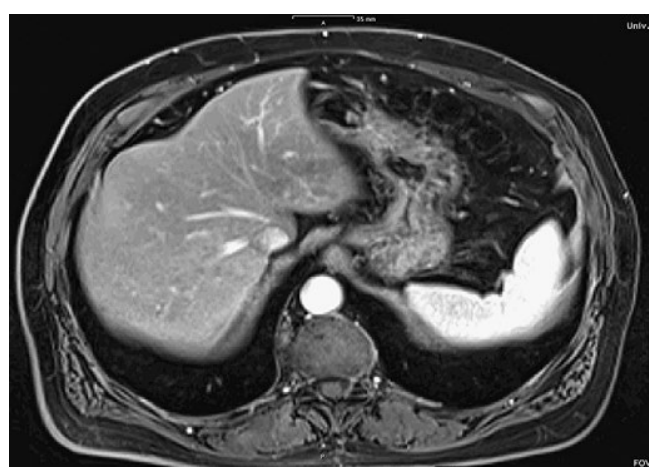

Fig. 7. MRI scan: no residual detectable metastases.

To discover genetic features which could potentially explain this exceptional response, fresh frozen and paraffin embedded tumor tissue was dissected for DNA isolation and panel sequencing of 710 cancer related genes (performed by CeGaT, Tübingen, Germany). Comparison of sequencing results with the TCGA database revealed only two previously described mutations: a frameshift deletion in NOTCH2 (p.P6Rfs $\left.{ }^{\star} 27\right)$ and a single nucleotide variant in tumor suppressor TP53 (missense mutation p.P72R). None of the mutations has been previously associated with therapy response to trastuzumab.

\section{DISCUSSION}

Our case report demonstrates that complete remission of a metastatic OGJ adenocarcinoma can be achieved with trastuzumab monotherapy. It needs to be noted that the patient initially received a combination chemotherapy, which resulted in a PR after first radiological restaging. However, a major tumor burden including liver metastasis was radiologically detectable when the treatment was reduced to trastuzumab monotherapy. The patient remained in SD for almost two years before achieving CR. There is no available data on synergistic effects of chemotherapy and trastuzumab, but regarding the initial PR under chemotherapy, we are unable to rule out beneficial effects resulting from combination treatment. Cardiac dysfunction is a major adverse effect of trastuzumab, but despite the long-lasting treatment, we did not observe trastuzumab-induced cardiotoxicity (TIC). A retrospective review of clinical trials using trastuzumab demonstrated an incidence of $3-7 \%$ for TIC for trastuzumab as a single treatment agent [7]. In a prospective clinical study, lower rates of TIC in gastric cancer patients treated with combination of chemotherapy and trastuzumab were reported compared to clinical trials in BC, indicating a cumulative cardiotoxic effect depending on the co-administered cytostatic medication [8].

The guidelines of the European Society for Molecular Oncology (ESMO) recommend a cisplatin-based regimen for palliative chemotherapy either with or without trastuzumab depending on HER2 status [9]. Since several clinical studies showed non-inferiority of oxaliplatin compared to cisplatin in conjunction with lower toxicity $[10,11]$, oxaliplatin-based therapies such as the FLOT regimen are preferred. FLOT chemotherapy regimen showed clinical activity and tolerable toxicity in clinical trials and therefore emerged as an option for first-line treatment in metastatic gastric cancer [12], but improved survival rates of this triplet therapy is also associated 
with higher toxicity [13]. This is mirrored in our case report, as the chemotherapy was discontinued due to CTC grade 3 adverse events after initial radiological response. Interestingly, Homann et al. [14] showed a pathological CR after preoperative FLOT therapy without antibody treatment in 8 of 46 patients, indicating a high activity of FLOT combination therapy. All 8 patients with CR had an intestinal type adenocarcinoma as the patient in our case report [14]. Whether these patients would have remained in complete remission under long-term treatment without surgery is unclear.

Review of the literature revealed two cases of long-term complete remission of metastatic GC under combination chemotherapy [15]. Both cases received docetaxel, 5-FU, leucovorin and cisplatin after metastatic relapse following gastrectomy. Complete remission was maintained for $40+$ and $54+$ months after discontinuation of chemotherapy.

Regarding BC, a small series of cases with complete response under trastuzumab treatment was reported, including a total of 17 patients with metastatic disease. Disease control in 12 out of these 17 patients was observed after discontinuation of anti-HER2 treatment [16-19], suggesting the existence of a subset of exceptional responders among HER2+ patients. Nevertheless, many patients who achieved complete response remained on maintenance therapy [19] due to lack of predictors of durable response.

In the ToGA trial, CR rate was reported to be $5 \%$ in patients who received chemotherapy plus trastuzumab versus $2 \%$ in the chemotherapy cohort, but duration of response and treatment of patients with $\mathrm{CR}$ are not reported [6]. One case report demonstrated initial PR and durable SD under trastuzumab in combination with FLO chemotherapy in a patient with OGJ adenocarcinoma despite the presence of only a minor fraction of HER 3+ tumor cells in the tissue [20]. Taken together, the results of the ToGA trial, the previously published case report and our data indicate that a subset of exceptional responders exists in GC/OGJ adenocarcinoma similar to BC. Further evidence for this observation is supported by the case report of a patient with HER2+ urothelial carcinoma, who also achieved complete and durable remission under treatment with trastuzumab and chemotherapy [21].

Sequencing of the tumor tissue of our patient showed no specific mutations which can sufficiently explain the exceptional response. TP53 is one of the best studied tumor suppressor genes, but there also is rising evidence for the role of NOTCH2 in progression and prognosis of gastric cancer [22, 23]. However, none of the genetic alterations have been found to be associated with therapy response to anti-HER2 treatment.

Although the molecular mechanisms for exceptional response remains unclear, data from a study of neoadjuvant administration of trastuzumab and paclitaxel \pm radiotherapy or anti-hormonal treatment in BC patients could link trastuzumab response with immunological changes such as decrease of immunosuppressive IL-10 and a polymorphism in the $F c$ receptor $(F c R)$ [24], suggesting a major role of immune system in trastuzumab mediated tumor control. Particularly, the FcR on macrophages and natural killer cells can initiate pro-apoptotic signals induced by release of cytotoxic molecules such as granzymes and perforines upon binding to membrane-bound antibodies on target cells, resulting in a lysis of the antibody-binding cell. This process is called antibodydependent cell-mediated cytotoxicity (ADCC) and there is evidence for the induction of ADCC by trastuzumab in vitro and in vivo in both BC and GC $[25,26]$. In addition, FcR can induce phagocytosis of trastuzumab-coated cells [27]. Thus, it would be of interest to analyze markers for the activity of the innate and adaptive immune system in a cohort of exceptional responders to trastuzumab.

Taken together, we present a case with durable, complete remission under trastuzumab monotherapy in a patient with metastatic OGJ adenocarcinoma even after discontinuation of treatment. Prospective clinical trials with an accompanying translational program could provide further insights into the underlying biological mechanism of an exceptional response in GC/OGJ adenocarcinoma, which would improve patient selection and reduce additional chemotherapy.

\section{CONCLUSION}

Trastuzumab is an important part of combination treatment for advanced GC and OGJ adenocarcinoma with high HER2 expression. Complete response can be achieved under trastuzumab monotherapy in exceptional responders. Further translational studies will be required to identify biomarkers for exceptional response to anti HER2 treatment.

\section{Conflicts of interest: None to declare.}

Authors' contribution: T.G., T.Z. conceived and wrote the manuscript. T.G., T.Z., N.S., S. B., J.B., N.H., J.Weers analysed and reviewed the data. J.Wilke, J.Weers, M.P.E. edited the manuscript, T.G. drafted the work. All authors critically revised the manuscript and approved the final version and agreed to be accountable for all aspects of the work.

Acknowledgements: The results shown here are in part based upon data generated by the TCGA Research Network (https://www.cancer. gov/tcga).

\section{REFERENCES}

1. Bray F, Ferlay J, Soerjomataram I, Siegel RL, Torre LA, Jemal A. Global cancer statistics 2018: GLOBOCAN estimates of incidence and mortality worldwide for 36 cancers in 185 countries. CA Cancer J Clin 2018;68:394-424. doi:10.3322/caac.21492

2. Tanner M, Hollmen M, Junttila TT, et al. Amplification of HER-2 in gastric carcinoma: association with Topoisomerase IIalpha gene amplification, intestinal type, poor prognosis and sensitivity to trastuzumab. Ann Oncol 2005;16:273-278. doi:10.1093/annonc/ mdi064

3. Van Cutsem E, Bang YJ, Feng-Yi F, et al. HER2 screening data from ToGA: targeting HER2 in gastric and gastroesophageal junction cancer. Gastric Cancer 2015;18:476-484. doi:10.1007/s10120-014-0402-y

4. Burstein HJ. The distinctive nature of HER2-positive breast cancers. N Engl J Med 2005;353:1652-1654. doi:10.1056/NEJMp058197

5. Mitri Z, Constantine T, O'Regan R. The HER2 Receptor in Breast Cancer: Pathophysiology, Clinical Use, and New Advances in Therapy. Chemother Res Pract 2012;2012:743193. doi:10.1155/2012/743193

6. Bang YJ, Van Cutsem E, Feyereislova A, et al. Trastuzumab in combination with chemotherapy versus chemotherapy alone for 
treatment of HER2-positive advanced gastric or gastro-oesophageal junction cancer (ToGA): a phase 3, open-label, randomised controlled trial. Lancet 2010;376:687-697. doi:10.1016/S0140-6736(10)61121-X

7. Seidman A, Hudis C, Pierri MK, et al. Cardiac dysfunction in the trastuzumab clinical trials experience. J Clin Oncol 2002;20:1215-1221. doi:10.1200/JCO.2002.20.5.1215

8. Soo Park J, Youn JC, Shim CY, et al. Cardiotoxicity of trastuzumab in patients with HER2-positive gastric cancer. Oncotarget 2017;8:6183761845. doi:10.18632/oncotarget.18700

9. Smyth EC, Verheij M, Allum W, et al. Gastric cancer: ESMO Clinical Practice Guidelines for diagnosis, treatment and follow-up. Ann Oncol 2016;27(suppl 5):v38-v49. doi:10.1093/annonc/mdw350

10. Cunningham D, Starling N, Rao S, et al. Capecitabine and oxaliplatin for advanced esophagogastric cancer. N Engl J Med 2008;358:36-46. doi:10.1056/NEJMoa073149

11. Al-Batran SE, Hartmann JT, Probst S, et al. Phase III trial in metastatic gastroesophageal adenocarcinoma with fluorouracil, leucovorin plus either oxaliplatin or cisplatin: a study of the Arbeitsgemeinschaft Internistische Onkologie. J Clin Oncol 2008;26:1435-1442. doi:10.1200/ jco.2007.13.9378

12. Al-Batran SE, Hartmann JT, Hofheinz R, et al. Biweekly fluorouracil, leucovorin, oxaliplatin, and docetaxel (FLOT) for patients with metastatic adenocarcinoma of the stomach or esophagogastric junction: a phase II trial of the Arbeitsgemeinschaft Internistische Onkologie. Ann Oncol 2008;19:1882-1887. doi:10.1093/annonc/mdn403

13. Wagner AD, Syn NL, Moehler M, et al. Chemotherapy for advanced gastric cancer. Cochrane Database Syst Rev 2017;8:CD004064. doi:10.1002/14651858.CD004064.pub4

14. Homann N, Pauligk C, Luley K, et al. Pathological complete remission in patients with oesophagogastric cancer receiving preoperative 5-fluorouracil, oxaliplatin and docetaxel. Int J Cancer 2012;130:17061713. doi:10.1002/ijc. 26180

15. Chan CH, Liu TW, Chen LT, Chang JY, Whang-Peng J. Longterm complete remission of metastatic gastric cancer after weekly docetaxel, $24 \mathrm{~h}$ infusion of high-dose 5-FU/leucovorin and cisplatin. J Gastroenterol Hepatol 2005;20:1470-1471. doi:10.1111/j.14401746.2005.03912.x

16. Viel E, Arbion F, Barbe C, Bougnoux P. Prolonged complete response after treatment withdrawal in HER2-overexpressed, hormone receptor-negative breast cancer with liver metastases: the prospect of disappearance of an incurable disease. BMC Cancer 2014;14:690. doi:10.1186/1471-2407-14-690
17. Murthy P, Kidwell KM, Schott AF, et al. Clinical predictors of long-term survival in HER2-positive metastatic breast cancer. Breast Cancer Res Treat 2016;155:589-595. doi:10.1007/s10549-016-3705-3

18. Hsieh A, Pittman K, Patterson WK, Townsend A. Can maintenance trastuzumab be stopped in patients with HER2-positive metastatic breast cancer? BMJ Case Rep 2015;2015:bcr2014207750. doi:10.1136/ bcr-2014-207750

19. Bishop AJ, Ensor J, Moulder SL, et al. Prognosis for patients with metastatic breast cancer who achieve a no-evidence-of-disease status after systemic or local therapy. Cancer 2015;121:4324-4332. doi:10.1002/ cncr.29681

20. Gaiser T, Hirsch D, Hofheinz RD. Good remission under HER2 blockade in an advanced carcinoma of the oesophagogastric junction with only focal HER2 overexpression. Z Gastroenterol 2017;55:866-871. doi:10.1055/s-0043-116493

21. Wezel F, Erben P, Gaiser T, et al. Complete and Durable Remission of Human Epidermal Growth Factor Receptor 2-Positive Metastatic Urothelial Carcinoma Following Third-Line Treatment with Trastuzumab and Gemcitabine. Urol Int 2018;100:122-125. doi:10.1159/000443280

22. Bauer L, Takacs A, Slotta-Huspenina J, et al. Clinical Significance of NOTCH1 and NOTCH2 Expression in Gastric Carcinomas: An Immunohistochemical Study. Front Oncol 2015;5:94. doi:10.3389/ fonc.2015.00094

23. Du X, Cheng Z, Wang YH, et al. Role of Notch signaling pathway in gastric cancer: a meta-analysis of the literature. World J Gastroenterol 2014;20:9191-9199.

24. Miolo G, Muraro E, Martorelli D, et al. Anthracycline-free neoadjuvant therapy induces pathological complete responses by exploiting immune proficiency in HER2+ breast cancer patients. BMC Cancer 2014;14:954. doi:10.1186/1471-2407-14-954

25. Kono K, Takahashi A, Ichihara F, Sugai H, Fujii H, Matsumoto Y. Impaired antibody-dependent cellular cytotoxicity mediated by herceptin in patients with gastric cancer. Cancer Res 2002;62:5813-5817.

26. Petricevic B, Laengle J, Singer J, et al. Trastuzumab mediates antibodydependent cell-mediated cytotoxicity and phagocytosis to the same extent in both adjuvant and metastatic HER2/neu breast cancer patients. J Transl Med 2013;11:307. doi:10.1186/1479-5876-11-307

27. Shi Y, Fan X, Deng H, et al. Trastuzumab triggers phagocytic killing of high HER2 cancer cells in vitro and in vivo by interaction with Fc $\gamma$ receptors on macrophages. J Immunol 2015;194:4379-4386. doi:10.4049/jimmunol.1402891 